Document downloaded from:

http://hdl.handle.net/10251/142900

This paper must be cited as:

Broatch, A.; Garcia Tiscar, J.; Roig-Villanueva, F.; Sharma, S. (07-2). Dynamic mode decomposition of the acoustic field in radial compressors. Aerospace Science and Technology. 90:388-400. https://doi.org/10.1016/j.ast.2019.05.015

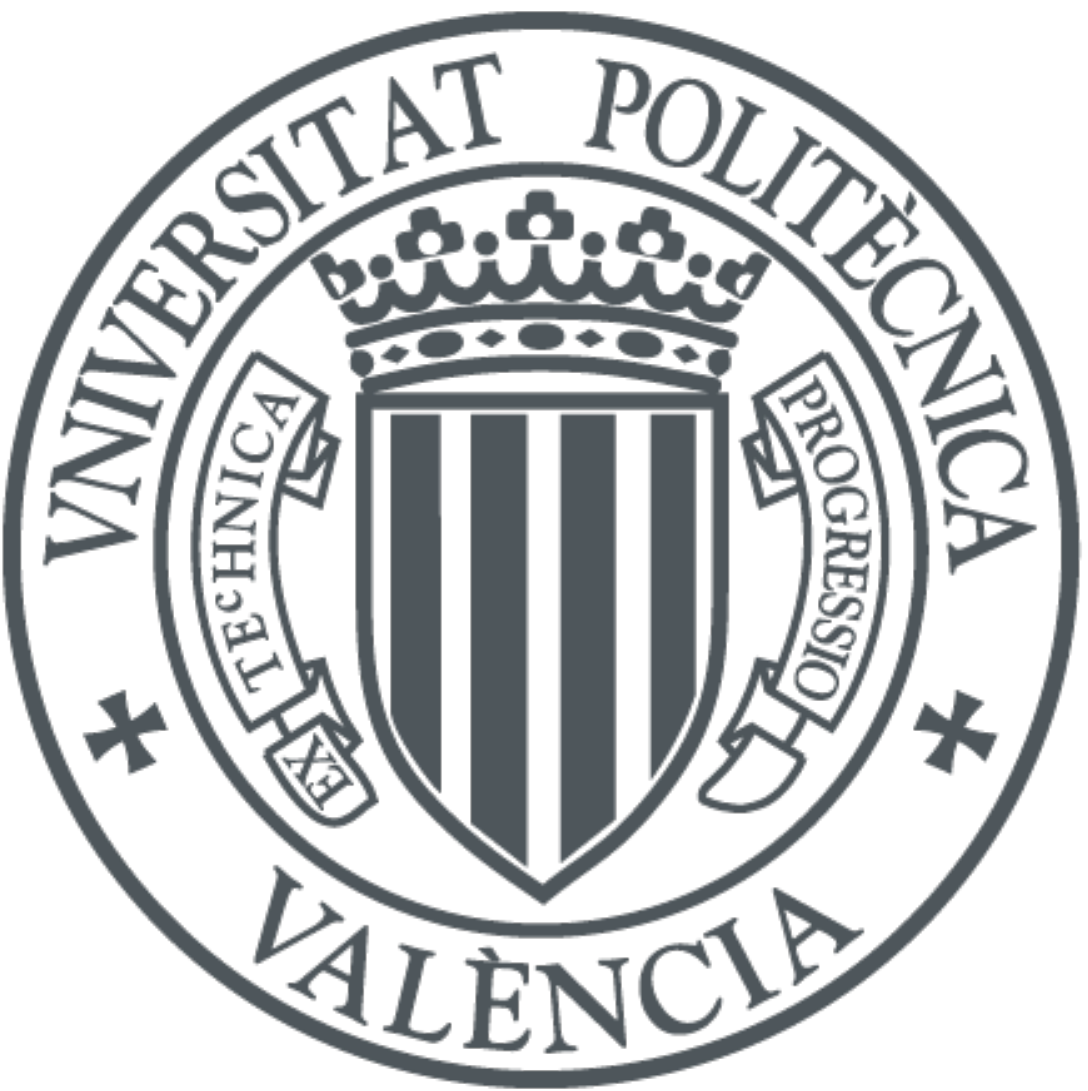

The final publication is available at

https://doi.org/10.1016/j.ast.2019.05.015

Copyright Elsevier

Additional Information 


\title{
Dynamic mode decomposition of the acoustic field in radial compressors
}

\author{
A. Broatch ${ }^{\mathrm{a}}$, J. García-Tíscar ${ }^{\mathrm{a}, *}$, F. Roig ${ }^{\mathrm{a}}, \mathrm{S} . \mathrm{Sharma}^{\mathrm{b}}$ \\ ${ }^{a}$ CMT - Motores Térmicos, Universitat Politècnica de València, Camino de Vera, 46022 Valencia, Spain \\ ${ }^{b}$ Turbocharger Research Institute, University of Huddersfield, Huddersfield, HDI 3DH, United Kingdom
}

\begin{abstract}
Widely recognized since the beginning of air travel as a major issue, noise reduction remains nowadays a pressing concern for all stakeholders in the aviation industry. While aeroengine compressors, specially at the approach phase, have been historically identified as a leading source of noise, most of the research has been conducted on compressors of the axial type. However, radial compressors are found in a wide array of applications: smaller business jets, helicopters, unmanned aerial vehicles (UAVs), auxiliary power units (APUs), turbochargers for reciprocating engines, etc. Owing to their geometrical particularities, radial compressors feature flow patterns that differ from their axial counterparts, leading to different acoustic performance but also opening the door for different optimization approaches. Yet, classical modal decomposition techniques focused on duct propagation may fail to reveal the complex interactions between geometry and flow features that act as noise sources. In this paper we apply, in addition to the classical approach, a data-driven Dynamic Mode Decomposition (DMD) to pressure data coming from a Detached Eddy Simulation (DES), in which we have experimentally validated the correct reproduction of the modal behaviour of the compressor, thus obtaining in-depth details of the link between flow phenomena and noise generation and transmission across the inlet and outlet ducts.
\end{abstract}

Keywords: Centrifugal compressors, Dynamic Mode Decomposition, Computer Fluid Dynamics, Aeroacoustics, Noise

\section{Introduction}

Since its very inception, noise has been one of the fundamental issues of commercial aviation-as an illustrative example, in 1935 Agatha Christie described the comfort of the Handley Page H.P.42 covering the Croydon-Le Bourget route by noting that 'The noise of the engines was very skilfully deadened. There was no need to put cotton wool in the ears' [1].

As aeronautical technology improved well beyond the strategic use of cotton wool and, especially, after the advances developed during WWII were implemented into civilian airliners, the plight of early air travel became a much more comfortable-and thus popular-experience.

Of special importance regarding noise and vibration was the introduction of the jet engine. While at first the jet exhaust itself deafened any other acoustic source, success of turbofan engines in reducing this issue uncovered a new problem for their manufacturers. The broadband jet flow noise was actually masking the compressor inlet discrete-frequency 'whine', which was now much more noticeable during the approach phase, thereby making aircraft noise an even worse problem in terms of sound quality [2].

It is in this historical context where the seminal work of Tyler and Sofrin [2] of Pratt \& Whitney Aircraft was presented. They sought to offer a complete analytical understanding of axial compressor acoustics, from the source pressure distribution to the duct propagation and ambient radiation. Their

*Corresponding author. CMT - Motores Térmicos, Universitat Politècnica de València, Camino de Vera, 46022 Valencia, Spain. jorgarti@mot.upv.es studies highlighted the role of rotating acoustic modes that were, at the time, regarded as phenomena of secondary importance [3].

While the analytical spinning mode approach has been used abundantly along the years in both numerical and experimental investigations of aircraft engine noise [4, 5], most of these investigations have been focused on axial turbomachinery. Studies regarding the modal decomposition of the acoustic field produced by radial machines have remained, on the other hand, relatively scarce in comparison [6].

As radial compressors feature flow phenomena that differ from those found in axial machines [7], it becomes necessary to investigate how these, in particular, relate with the generation and propagation of noise at different frequencies. While at design condition tonal noise at the Blade Passing Frequency (BPF), caused by the rotation of pressure patterns associated to the aerodynamic blade loading, behaves similarly in both kinds of compressor, stall and surge dynamics of radial machines are still less understood [8, 9].

Particularly, non-axisymmetric volutes that create pressure differences along the angular coordinate [10] cause nonuniformities in the flow field and have been found to influence the location of shock waves in the passages, leading edge spillages and tip leakage flow trajectories [11].

In turn, these unstable flow phenomena related to the geometric characteristics are linked to the spectral signature of the compressor noise [12]. This opens the door to geometry improvements that optimize both flow stability and acoustics, such as the use of hub [13] or shroud [14, 15] cavities. 


\section{Nomenclature}

List of symbols

A Modal amplitude (-)

$c_{p}$ Specific heat capacity at const. $\mathrm{p}\left(\mathrm{J} \mathrm{kg}^{-1} \mathrm{~K}^{-1}\right)$

$f$ Frequency (Hz)

$k_{x}$ Modal axial wavenumber (-)

$\dot{m}$ Mass flow rate $\left(\mathrm{kg} \mathrm{s}^{-1}\right)$

$p$ Pressure $(\mathrm{Pa})$

$R$ Duct radius (m)

$r$ Radial coordinate (m)

$T$ Temperature (K)

$t$ Time (s)

$x$ Axial coordinate $(\mathrm{m})$

$y^{+}$Non-dimensional boundary layer distance (-)

$\gamma$ Ratio of specific heats (-)

$\theta$ Azimuthal coordinate (rad)

$\kappa$ Modal radial wavenumber (-)

$\lambda$ DMD modal eigenvalue (-)
$\Phi$ DMD modal shape (-)

$\varphi$ Azimuthal modal distribution (-)

List of sub- and superscripts

0 Mean (flow variable)

co Cut-off (frequency)

in Inlet duct

m Azimuthal modal order

n Radial modal order

out Outlet duct

$\mathrm{T}$ Total or stagnation variable

List of abbreviations

BPF Blade Passing Frequency

DMD Dynamic Mode Decomposition

PSD Power Spectral Density
In this paper, a better understanding of the relationship between the radial compressor geometry, its unstable flow features, and the corresponding acoustic phenomena is sought by means of modal decomposition techniques applied to a numerical simulation of the system operating at the onset of the mild surge region where unstable flow features start to manifest.

\section{Theoretical background}

\subsection{Analytical spinning modes}

In order to facilitate the mathematical treatment of the compressor acoustics, Tyler \& Sofrin proposed [2] a modal decomposition of the acoustic field into rotating pressure patterns referred to as 'spinning modes'. Propagation of these analytical modes was studied by first considering simplified media such as thin rectangular ducts and narrow annular ducts which could be understood as 2D cases, and then an annular duct typical of axial turbomachinery.

However, this analysis primarily concerned tonal noise caused by the rotor only and by the rotor-stator interaction, disregarding other broadband noises of aerodynamic origin. In particular, a steady aerodynamic loading of the blades rather than unstable vortex-shedding or partially detached regimes was considered. This steady loading was found to create 'lobed' circumferential distributions of pressure.

Several authors [3, 16-18] have developed this analytical model over the years, showing that in the case of a uniform circular duct of radius $R$ with axially uniform mean flow at Mach $M_{0}$, it is indeed possible to write the well-known convected wave equation

$$
\left(\frac{\partial}{\partial t}+M_{0} \frac{\partial}{\partial x}\right)^{2} p=\nabla^{2} p
$$

in such a way that a solution for the pressure field can be obtained as a superposition of Tyler-Sofrin spinning modes in polar coordinates $(r, \theta, x)$ and time $t$ :

$$
p(r, \theta, x, t)=\Re\left\{\sum_{m}^{\infty} \sum_{n}^{\infty} A_{m n}^{ \pm} J_{m}\left(\kappa_{m n} \frac{r}{R}\right) e^{i m \theta} e^{-i \omega t} e^{i k_{x_{m n}}^{ \pm} x}\right\}
$$

Subscript $m \in \ldots,-2,-1,0,1,2, \ldots$ refers to the azimuthal order of the mode, this is, the number of wavelenghts of the 'lobed' distribution in the circumferential direction. In a compressor without stator blades it is expected that the main orders are those of $m=h n_{b}$ with $h \in 1,2, \ldots$ and $n_{b}$ being the number of rotor blades, this is, those corresponding to the Blade Passing Frequency (BPF) and its harmonics.

On the other hand, subscript $n \in 0,1,2, \ldots$ refers to the radial order, this is, the number of zeros across the radius. Alternatively, $m$ and $n$ can be seen, respectively, as the number of nodal lines and nodal circles within a cross-section, with the special case $(m, n)=(0,0)$ representing a plane wave.

Inspecting Eq. 2, $A_{m n}^{ \pm}$is seen to represent the unknown amplitude of each $(m, n)$ mode in both positive and negative axial propagation direction. $J_{m}$ is the Bessel function of the first kind and order $m$, whereas $\kappa_{m n}$, the radial wavenumber, satisfies the imposed boundary condition of an acoustic hard wall at the outer radius $r=R$ by requiring the first derivative of the Bessel function $J_{m}^{\prime}\left(\kappa_{m n}\right)$ to be zero. An illustrative visual example of this requirement in connection with the $(m, n)$ order of the modal shapes can be found in Figs. 2 and 3 of Eriksson's 1980 paper [19].

Whereas the first two exponential terms $e^{i m \theta}$ and $e^{-i \omega t}$ in eq. 2 are easily understood as related to the azimuthal 'lobed' structure of the mode and the driving frequency $\omega=2 \pi f$ of the mode spinning, it is important to carefully consider the axial wavenumber found in the last exponential term, as it is 
related to the propagation of the signal along the duct:

$$
k_{x_{m n}}^{ \pm}=\frac{k}{1-M_{0}^{2}}\left(-M_{0} \pm \sqrt{1-\left(1-M_{0}^{2}\right)\left(\frac{\kappa_{m n}}{k R}\right)^{2}}\right)
$$

Here, $k=\omega / c_{0}$ is the acoustic wavenumber, with $c_{0}$ being the speed of sound, and the \pm superscript again indicating that a certain mode can propagate in either axial direction with a corresponding $A_{m n}^{ \pm}$amplitude. Analyzing this expression, one can identify a specific 'cut-off' frequency at which the argument of the square root becomes zero:

$$
f_{m n}^{c o}=\kappa_{m n} \frac{c_{0}}{2 \pi R} \sqrt{1-M_{0}^{2}}
$$

Taking into account the role of $k_{m n}^{ \pm}$in Eq. 2, it can be seen that for a driving frequency $f<f_{m n}^{c o}$, the downstream propagation term $\Re\left\{e^{i k_{x_{m n}}^{+} x}\right\}$ has a decaying component $e^{-\mathfrak{S}\left\{k_{x_{m n}}^{+}\right\} x}$, and thus mode $(m, n)$ is said to be 'cut-off', damped, or evanescent, since it will vanish at a certain distance from the source.

On the other hand, for values of the driving frequency $f>f_{m n}^{c o}$ the mode is 'cut-on', as the real part of the downstream propagation term in Eq. 2 takes oscillatory values $\cos \left(k_{x_{m n}}^{+} x\right)$ and thus the mode propagates unattenuated. A visual representation of the physical meaning of the cut-off criterion can be found in Fig. 2 of Morfey's 1964 paper [3].

An example of the pressure distribution of a Tyler-Sofrin mode is given in Fig. 1, where mode $(6,1)$ according to Eqs. 2 and 3 is represented. The rest of the parameters have been arbitrarily chosen so that $f>f_{6,1}^{c o}$ and thus the mode propagates along the duct. The physical meaning of the cross-section pressure pattern in relation to the aerodynamic loading of the compressor blades is apparent.

Thus, in order to find the unknown modal amplitudes $A_{m n}^{ \pm}$, one can either conduct experimental measurements or numerical simulations of $p(t)$ at different locations and then perform a least-squares fitting following the procedure recommended by Moore [20]. In his experimental rig, a single microphone was motorized so it could reach different radial and circumferential positions at the measurement plane.

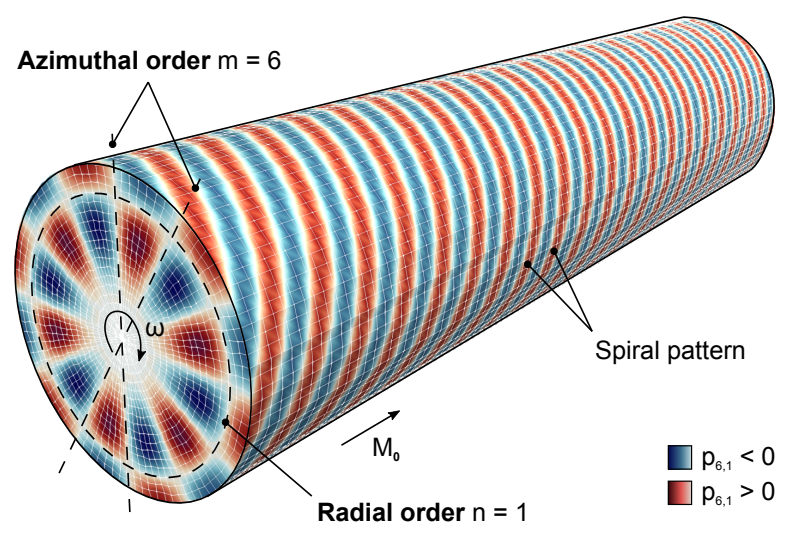

Figure 1: Example of the Tylor-Sofrin mode $(6,1)$ numerically evaluated with arbitrary values chosen so that $f>f_{6,1}^{c o}$
Later, authors such as Raitor \& Neise [6] implemented these techniques in the experimental characterization of centrifugal fans and turbocompressors. Most recently, Limacher, Banica et al. investigated the sound field of centrifugal turbochargers through the least-square fitting of select blade passing frequencies to Tyler-Sofrin modes from both numerical [21] and experimental [22] points of view.

While these works successfully demonstrate that the pressure distribution of the expected Tyler-Sofrin modes $\left(h n_{b}, 0\right)$ is found in the measurement sections for the frequency corresponding to the BPF tone and its harmonics, this approach is not without drawbacks, especially in centrifugal compressors.

Besides the restriction of the shape of the modes themselves, which must adhere to Eq. 2, several assumptions are used. Excitation is introduced in an 'actuator disk' manner, and then equally propagated up and downstream. Especially the latter is questionable in centrifugal compressors, in which the outlet, contrary to the inlet, does not directly 'see' the rotating blade pressure distribution as the perturbations must first travel through the complex flow in the volute.

Furthermore, while uniform, unidirectional flow is assumed, it is known that between the onset of the marginal surge conditions to the deep surge limit, recirculating backflow at high temperature is present in the inlet section [23]. Also, while evanescent modes may not fully propagate beyond a short distance, both these local phenomena near the compressor may induce vibroacoustic excitation on the pipes, often made of rigid plastic materials to save weight and cost, and thus still radiate noise.

It can be seen in the aforementioned studies [6, 21, 22] that when noise phenomena other than BPF harmonic tones are decomposed in this way, a large number of mode orders become excited, this is, a large sum along the $m$ and $n$ orders is required in order to explain the observed pressure pattern at the measured cross-section.

A large sum of modes with similar amplitudes $A_{m n}^{ \pm}$makes difficult to understand the spatial distribution of the phenomenon, indicating that the actual pressure pattern is not particularly similar to any specific mode. Thereby, it is possible that the Taylor-Sofrin analytical model may not be the optimum decomposition in these particular situations.

\subsection{Dynamic Mode Decomposition}

A very different alternative to the analytical solution lies in the use of data-driven numerical decompositions, such as the classical Proper Orthogonal Decomposition (POD) [24] which was pioneered by Lumley in 1967 for detection of spatial coherent patterns in turbulent flows [25] and has since been used widely in aeronautical research [26-30].

A recent alternative, better suited for the identification of acoustic phenomena at particular frequencies, is the Dynamic Mode Decomposition (DMD) [31], a technique which aims to group coherent spatial features into modes of a single temporal frequency [32, 33]. It has been used in recent investigations of, for instance, cavity flow [34], airfoil buffeting 
[35], fan [36] combustion [37, 38] and landing gear [39] noise, and turbocharger acoustics [40,41], among others.

This decomposition method is based in estimating the eigenmodes and eigenvectors associated to the Koopman operator of a given system, an infinite-dimensional but linear operator which is used to describe the dynamics of finitedimensional but non-linear systems [42]. DMD eigenvalues obtained in this way contain a single frequency, together with the growth or decay rates associated to their associated mode. This allows the identification of transient modes that may be short-lived or low-energetic in nature but still coherent [43].

In general, the evolution of a field such as in this case the pressure field $p(r, \theta, x, t)$ can be described by a temporal sequence $\mathbf{V}_{1}^{N}$ of vectors $\mathbf{v}_{t}(\mathbf{x})$, each one containing the pressure data at a certain spatial position $\mathbf{x}=\{r, \theta, x\}$ and at a certain discrete time step $t \in 1,2, \ldots, N$ :

$$
\mathbf{V}_{1}^{N}=\left\{\mathbf{v}_{1}, \mathbf{v}_{2}, \ldots, \mathbf{v}_{N}\right\}
$$

This representation fits naturally flow field characterizations offered by experimental techniques such as Particle Image Velocimetry (PIV), as well as those obtained through Computer Fluid-Dynamics (CFD) simulations. In both cases data is obtained as several 'snapshots' of the flow variables (velocity, pressure, density, species, etc.), that could be put intuitively in column vector form, and then assembled in a matrix $\mathbf{V}$ describing the overall evolution of the selected variable.

By following the original procedure introduced by Schmid [31], DMD can be applied to the snapshot matrix $\mathbf{V}_{1}^{N}$ of Eq. 5 . If we assume that the snapshots $\mathbf{v}_{i}$ which conform the matrix are linearly related by an unknown matrix A:

$$
\mathbf{v}_{i+1}=\mathbf{A} \mathbf{v}_{i}
$$

Then the eigenvalues and eigenvectors of this matrix will characterize the evolution of the flow. Even if the system is non-linear, this will provide a linear tangent approximation of the flow evolution. We can combine Eqs. 5 and 6 to write:

$$
\mathbf{V}_{2}^{N}=\mathbf{A V}_{1}^{N-1}
$$

While some authors solve this eigensystem by QR decomposition of the associated companion matrix [32, 44, 45], a more robust alternative makes use of the Singular Value Decomposition (SVD) $\mathbf{V}_{1}^{N-1}=\mathbf{U} \Sigma \mathbf{W}^{\mathrm{T}}[31,33,46,47]$. Plugging the SVD decomposition into Eq. 7 we obtain:

$$
\mathbf{V}_{2}^{N}=\mathbf{A U} \Sigma \mathbf{W}^{\mathrm{T}}
$$

We now build a matrix $\widetilde{\mathbf{S}}$, which is defined exclusively in terms of known matrices but is constructed in a way that ensures matrix similarity with A:

$$
\widetilde{\mathbf{S}} \triangleq \mathbf{U}^{\mathrm{T}} \mathbf{V}_{2}^{N} \mathbf{W} \boldsymbol{\Sigma}^{-1}=\mathbf{U}^{\mathrm{T}} \mathbf{A U}
$$

As a consequence of this similarity, the eigenvalues $\lambda_{i}$ of the reduced-size $\widetilde{\mathbf{S}}$ match those of the larger A, with the DMD modal shapes being computed by mapping the eigenvector matrix $\mathbf{Y}$ of $\widetilde{\mathbf{S}}$ into the non-reduced space through $\mathbf{U}$, which we can recognize as being the POD basis of $\mathbf{V}_{1}^{N-1}$ :

$$
\Phi=\mathbf{U Y}
$$

However, since many numerical routines normalize the resulting eigenvectors, it is necessary to recover the modal amplitudes $\alpha_{i}$, which we can intuitively do by solving the reconstructed flow field multiplied by the unknown amplitudes against, for instance, the first snapshot of the flow [48]:

$$
\mathrm{V}_{1}=\Phi \alpha \Longrightarrow \alpha=\Phi^{-1} \mathrm{~V}_{1}=\mathrm{Y}^{-1} \mathrm{U}^{*} \mathrm{~V}_{1}
$$

Where we have taken advantage of the fact that $\mathbf{U}$ is unitary and thus its conjugate transpose $\mathbf{U}^{*}$ is also its inverse, in order to solve the system by inverting $\mathrm{Y}$ instead of the higher order $\boldsymbol{\Phi}$. Now, the full evolution of the flow described by the snapshot matrix at discrete time steps $t_{k}$, can be reconstructed by the linear superposition of the DMD modes:

$$
\mathbf{V}\left(\mathbf{x}, t_{k}\right)=\Re\left\{\sum_{i=1}^{N-1} \boldsymbol{\Phi}_{i}(\mathbf{x}) \alpha_{i} \lambda_{i}^{k-1}\right\}=\Re\left\{\boldsymbol{\Phi} \mathbf{D}_{\text {iag }}(\boldsymbol{\alpha}) \mathbf{V}_{\text {and }}(\boldsymbol{\lambda})\right\}
$$

Here, $\mathbf{D}_{\text {iag }}(\boldsymbol{\alpha})$ is the diagonal matrix of modal amplitudes and $\mathbf{V}_{\text {and }}(\boldsymbol{\lambda})$ is the Vandermonde matrix of the eigenvalues. Furthermore, from the eigenvalues we can recover the single frequency associated to each mode, by taking into account the time step $\Delta t$ between each consecutive snapshot:

$$
f_{i}=\frac{\omega_{i}}{2 \pi}=\frac{\mathfrak{I}\left\{\ln \left(\lambda_{i}\right)\right\}}{2 \pi \Delta t}
$$

Finally, the computed modes need to be ranked in importance. While the most direct approach consists in computing the energy of the eigenvectors augmented by the amplitude coefficients [48], Kou \& Zhang [49] have recently proposed a simple criterion which integrates the influence of each dynamic mode on the whole sampling space:

$$
E_{i}=\sum_{j=1}^{N}\left|\alpha_{i} \lambda_{i}^{j-1}\right|\left\|\Phi_{i}\right\|_{F}^{2} \Delta t
$$

Since in this work the focus is put into the steady operation of the compressor, short-lived evanescent modes are not expected nor of interest for the objective of isolating the most energetic and relevant modes. Thus, the Kou \& Zhang criterion will be used to rank the DMD modes.

\section{Numerical model}

The CFD model used in this work to obtain suitable flow field snapshots has its basis in the work of Broatch et al. [5052], which is briefly described herein. The impeller with 6 full and 6 splitter blades of a radial compressor was digitalized along with its vaneless diffuser and the compressor volute. Backplate clearance and tip gap were considered as well in the model. 
However, as it was estimated that adding the complete experimental rig ducts would have increased the computational cost by ten times [50] so five-diameters-long inlet and outlet straight ducts were used to complete the computational domain. This domain will constitute the baseline against which the improvements in this investigation will be assessed.

The numerical configuration followed the criteria used by Mendonça et al. [53]. A computational mesh of 9.5 million polyhedral cells was employed providing $y^{+}$values close to unity at the impeller. A segregated solver was selected to perform a detached-eddy simulation (DES) with a SST $k-\omega$ turbulence model. Outlet pressure and inlet mass flow boundary conditions were set, and the chosen time discretization $\Delta t=1.046 \times 10^{-6} \mathrm{~s}$ rendered $1^{0}$ impeller rotation per time step, in accordance with the sensitivity analysis performed by Navarro [54]. Heat transfer with surroundings was neglected, as it was justified [55] that it did not influence the compressor global variables in the chosen operating conditions.

The described model was validated at three working points: one close to the best efficiency point (BEP), other at maximum pressure ratio (MPR) and one close to deep surge [52]. Compressor global variables were predicted with a relative error below 3\%. Inlet and outlet in-duct pressure spectra were calculated and compared with experimental data.

While certain features were correctly captured by the CFD model, such as BPF tonal noise, other trends were not well reproduced, particularly broadband elevations at higher frequencies. There were as well a number of traits in the CFD spectra not seen in the experimental ones, like low-frequency ripples in both ducts. These abnormal features were associated with the presence of standing waves caused by the reduced duct length [50].

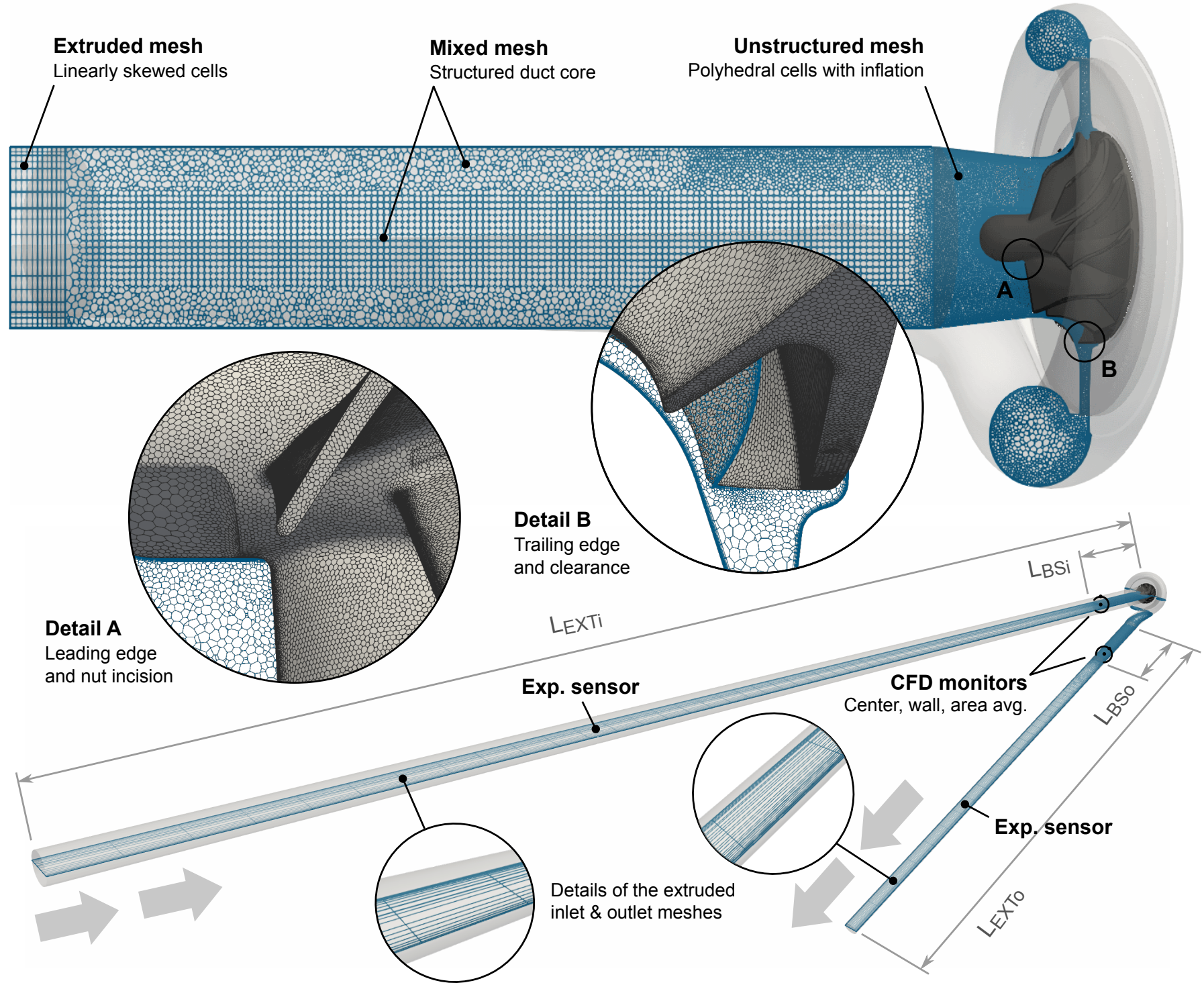

Figure 2: Illustration of the full numerical domain, highlighting the extended duct lengths (EXT) versus the baseline (BS) ones, and including details of the meshing strategy and the location of both numerical monitors and experimental sensors. 


\subsection{Extended model}

To tackle these issues, a beamforming wave decomposition technique [56] was applied to remove the reflected components and consider only frequency content originated at the compressor. The resulting spectra presented improved agreement with experimental data, but the frequency range available for analysis was reduced as a Nyquist-type criterion imposed by the distance between pressure sensors has to be considered.

Most importantly regarding the present investigation, even when reliable pressure spectra can be extracted from the model data by performing beamforming pressure decomposition, the spurious frequency content is still present in the predicted flow field whose cell values are used to perform post-processing techniques like modal decomposition.

In the present investigation, the CFD model was built from the described setup, extending the inlet and outlet ducts to the length of the experimental rig, in order to alleviate the aforementioned issues. The new CFD domain can be seen in Fig. 2. The new ducts were meshed following a strategy of extruding the cells at the ends of the original ducts in the axial direction.

Cell lengths were increased in a geometric progression from the original size at the end of the baseline ducts to a length of $80 \mathrm{~mm}$ at the end of the extended ones, as Fig. 2 shows. The structured core mesh of the previous model was kept in order to reduce cell count, with unstructured polyhedral cells comprising the rest of the domain, save for the inflation layers at the surfaces.

In consequence, the large regions added to the domain were meshed with a low cell count of $90 \times 10^{3}$ cells for the inlet duct and $80 \times 10^{3}$ for the outlet, increasing only by $1.8 \%$ the total number of cells. The resulting mesh keeps $y^{+}$values close to unity near the impeller while at the new ducts the values are lower than 3.3 for the inlet and 2.5 for the outlet, enabling the low-Reynolds resolution of the boundary layer.

Another useful feature of the new mesh is the numerical damping it introduces. The fine mesh between the compressor wheel and the monitors allows all the frequencies of interest to be properly resolved, while the coarse mesh between the boundaries and the monitors dampens the reflected frequency content. In particular, the maximum cell length of $80 \mathrm{~mm}$ attenuates reflected frequencies above $1200 \mathrm{~Hz}$, considering that wavelengths exceeding four times the cell length are not properly captured by the mesh.

\subsection{Experimental validation}

The CFD model described above grants access to useful information about the fluid field which can be very difficult to measure at the experimental rig. However, it is imperative to assess how well the model reproduces the real flow field before drawing conclusions from CFD data. In this section the methodology of validation is described.

Two sets of data are compared: first compressor global variables are calculated, and then spectra of pressure signals at inlet and outlet ducts are studied, following the methodology presented by Broatch et al. [50].

\subsubsection{Validation of global variables}

Specific work, isentropic efficiency and total to total compression ratio, are considered to assess how accurately CFD predicts the compressor working point.

$$
\begin{aligned}
W_{u} & =\frac{\dot{W}}{\dot{m}}=\frac{\Omega \tau}{\dot{m}}=c_{p}\left(T_{\text {out }, T}-T_{\text {in }, T}\right) \\
\eta_{s} & =\frac{\dot{W}_{s}}{\dot{W}}=\frac{T_{\text {in }, T}\left(\Pi_{T T}^{\frac{\gamma-1}{\gamma}}-1\right)}{T_{\text {out }, T}-T_{\text {in }, T}} \\
\Pi_{T T} & =\frac{p_{\text {out }, T}}{p_{\text {in }, T}}
\end{aligned}
$$

The chosen simulation working point is the one of maximum compression ratio (null isospeed slope) for the $\Omega=160$ krpm characteristic line, which corresponds to an air mass flow $(\dot{m})$ of $77 \mathrm{~g} / \mathrm{s}$. The location of the selected point in the compressor map is available in ref. [50]. This specific point was chosen as it marks the beginning of the mild surge region in which flow instabilities start affecting the performance of the compressor, reducing efficiency and introducing additional noise phenomena. The position of inlet and outlet pressure and temperature sensors is the same as in the experimental rig. A comparison can be seen in Table 1 .

Table 1: Validation of global variables

\begin{tabular}{lccc}
\hline & $W_{u}[\mathrm{~kJ} / \mathrm{kg}]$ & $\Pi_{\mathrm{TT}}[-]$ & $\eta_{s}[\%]$ \\
\hline Experimental data & 112.0 & 2.24 & $67.8 \%$ \\
Extended CFD model & 111.9 & 2.21 & $66.8 \%$ \\
\hline Relative error & $0.07 \%$ & $1.35 \%$ & $1.46 \%$ \\
\hline
\end{tabular}

The agreement between CFD and experimental global variables is very good, with relative errors no larger than $1.5 \%$. However, since agreement in terms of global variables does not guarantee a proper prediction of local flow field [57], hence validation of pressure spectra was carried out.

\subsubsection{Validation of pressure spectra}

In the experimental rig, in-duct pressure signals are captured by two arrays of three flush-mounted pressure sensors, spotted in the inlet and outlet ducts as seen in [50]. In the original CFD model the monitors were placed closer to the compressor, as the ducts were shorter than their experimental rig counterparts. In the extended duct model, the original monitor location was kept because of the mesh coarsening, which prevented frequency content of interest to be appropriately captured. The distance between experimental and CFD arrays at the inlet is above $1.5 \mathrm{~m}$ which helps explaining the small offset in PSD amplitude found in Fig. 3. 

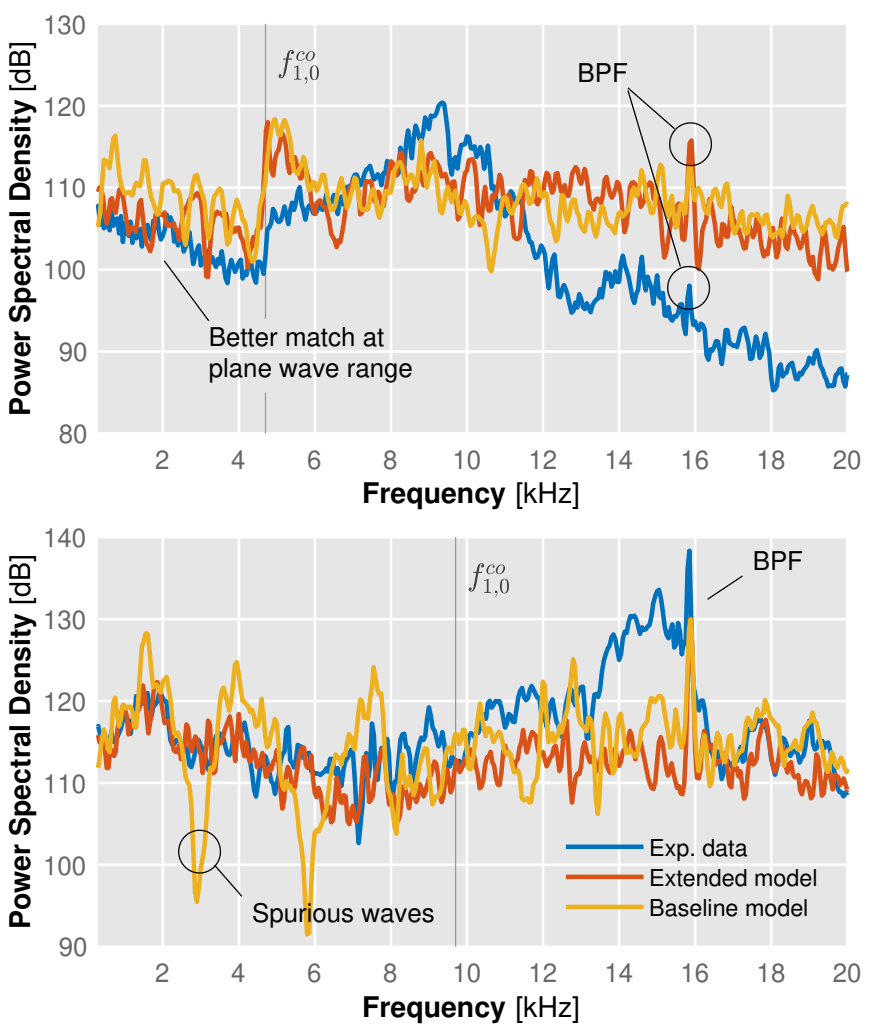

Figure 3: PSD of baseline and extended models vs. experimental data for both inlet (top plot) and outlet (bottom plot).

Experimental signals were captured during $1 \mathrm{~s}$, and CFD data was recorded during $42 \mathrm{~ms}$-corresponding to $110 \mathrm{im}$ peller revolutions-after a steady state was reached in terms of global variables. In the calculation of PSD, Welch's average was used [58]. Blocks with $50 \%$ overlap and Hamming window function were selected, and the number of blocks was aimed at obtaining a frequency resolution of $50 \mathrm{~Hz}$.

Inlet PSD calculated from the raw pressure signal captured by the first sensor of the array can be seen in Fig. 3. The main features of the experimental spectrum are an initial decay until $4.7 \mathrm{kHz}\left(f_{1,0}^{c o}\right)$, a broadband elevation from $4.7 \mathrm{kHz}$ to $12 \mathrm{kHz}$ and a decrease in amplitude from $12 \mathrm{kHz}$ onwards.

The CFD extended model is able to reproduce the initial decay in the plane wave range, and the increase in amplitude after $f_{1,0}^{c o}$, but it does not capture the broadband elevation; instead, amplitude decreases slowly from $14 \mathrm{kHz}$ onwards. Yet, the model is able to capture the blade passing frequency tone at about $16 \mathrm{kHz}$. Of course, above $f_{1,0}^{c o}$ the raw PSD signals differ as $3 \mathrm{D}$ acoustic modes start propagating along the duct, affecting the sensors differently due to their different spatial locations.

Meanwhile, the relevant traits that the outlet PSD presents are broadband elevations from $750 \mathrm{~Hz}$ to $2500 \mathrm{~Hz}$ and from $13 \mathrm{kHz}$ to $16 \mathrm{kHz}$, and the BPF tone at $16 \mathrm{kHz}$. From the above, CFD model is not able to capture the 13 to $16 \mathrm{kHz}$ hump, although it follows quite accurately the experimental spectrum elsewhere. In conclusion, the new CFD model was able to reproduce the acoustic field measured at the experimental rig, except for the above broadband elevations in both inlet and outlet spectra that occur beyond the plane wave range.

Comparing the extended domain against the baseline one, it can be seen in Fig. 3 how, at the inlet, ripples in the $0-5 \mathrm{kHz}$ range have been sufficiently attenuated, a fact which can be attributed to the alleviation of the standing waves due to the duct length increase. The offset between experimental and CFD spectrum in that frequency range has been reduced as well. At the outlet remarkable improvements are found in the low frequency range, where the new model follows significantly better the experimental measurements.

In conclusion, the low frequency spectrum was greatly improved, particularly at the outlet, being the removal of the standing waves the main contribution of the ducts extension. The high frequency spectrum obtained with both models is the same. Solving the problem of spurious reflected frequency content, due to the duct extension and the stretched mesh, allowed for the safe use of post-processing techniques like modal decomposition, from raw pressure data.

\subsubsection{Validation of acoustic modes propagation}

Even if raw signals above the plane wave range cannot be directly compared, further validation of the CFD model can be accomplished precisely by analyzing this $3 \mathrm{D}$ behaviour. Figure 4 depicts spectra of the pressure signals captured by three monitors in the same axial position: two point monitors
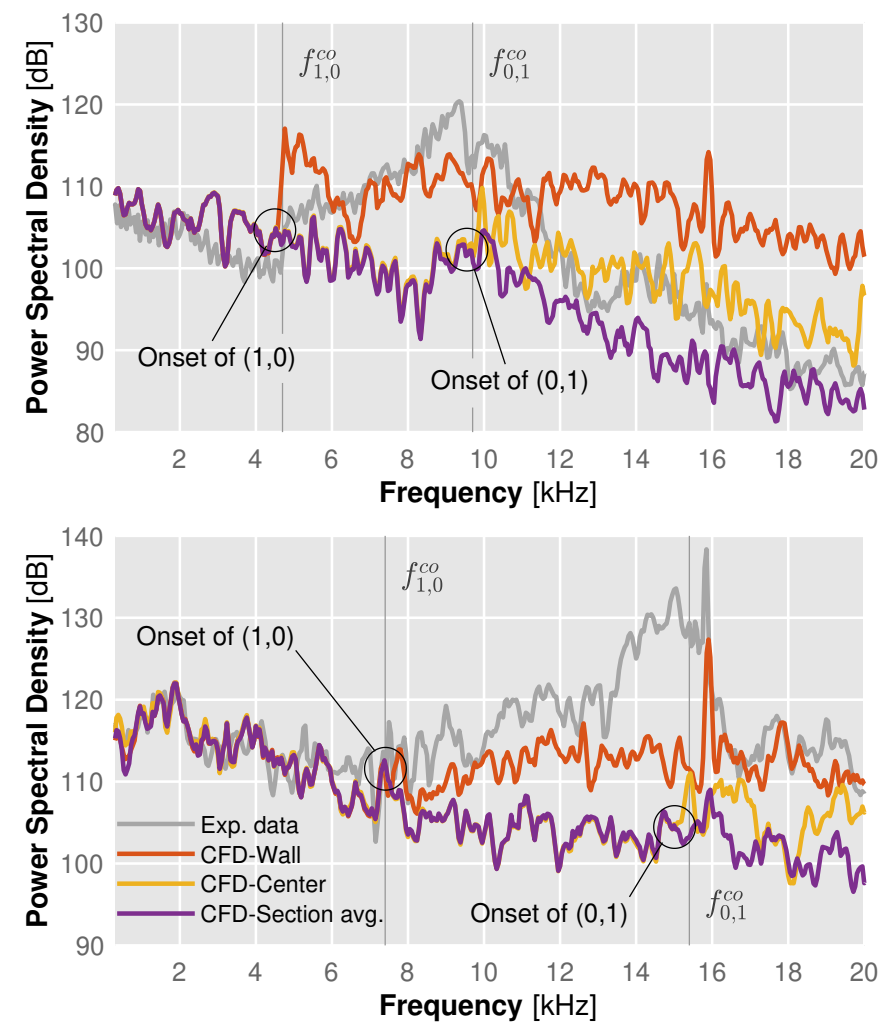

Figure 4: $P S D$ of different CFD monitors vs. experimental data for both inlet (top plot) and outlet (bottom plot), including theoretical Tyler-Sofrin cut-off frequencies. 
located at the duct axis and next to the wall, and a cross section monitor. As per Eq. 4, the first asymmetric mode $(1,0)$ and the first radial mode $(0,1)$ should start propagating at $f_{1,0}^{c o}=4.7 \mathrm{kHz}$ and $f_{0,1}^{c o}=9.7 \mathrm{kHz}$ in the case of the inlet duct and at $7.4 \mathrm{kHz}$ and $15.4 \mathrm{kHz}$ in the case of the outlet duct.

Both $f_{1,0}^{c o}$ frequencies can be clearly seen in Fig. 4 to accurately match the point where the numerically-predicted spectrum of the wall monitor begins to differ from that of the center and area-averaged monitors, for both inlet and outlet ducts. This is explained in light of the modal shape of the $(1,0)$ Tyler-Sofrin mode featuring a nodal line through the center, thereby relieving the center monitor from the oscillations of this mode which nonetheless affect the wall monitor.

A similar mechanism explains the departure of the prediction by the center and area-averaged monitors at the onset of the mode $(0,1)$. Whereas for $n=0$ modal shapes the center monitor lied in a nodal point, 'filtering out' the additional asymmetric mode oscillations being superimposed over the averaged pressure, at the cut-on frequency of the first circular mode $f_{0,1}^{c o}$ the center monitor lies in the point of maximum modal amplitude, and thus its readings start diverging from the averaged cross-section data in both ducts.

Crucially, this demonstrated that the simulated flow field was accurately reproducing 3D acoustic modal phenomena above the plane wave range, and thus a data-driven modal decomposition of such flow field was viable and representative of the real-world modal behaviour of the centrifugal compressor.

\subsection{Data preparation for decomposition}

Thus, in order to apply the modal decomposition techniques outlined in section 2 , the full pressure field data was exported to text files containing column vectors for the cell centroid coordinates $[\mathbf{x}, \mathbf{y}, \mathbf{z}]$ and their corresponding static pressures $\mathbf{p}$. In order to optimize network transfer and disk space, data was exported only after each five time steps, in order to obtain a sampling frequency of $190 \mathrm{kHz}$, more than enough to apply the Nyquist criterion in order to isolate acoustically-relevant information.

However, since the cells of the impeller and diffuser regions are rotating, the spatial coordinates of each pressure snapshot do not remain constant. As the decomposition procedures require constant spatial coordinates in order to provide a meaningful result, a previous step needs to be performed on the raw data.

In this preliminary step, a single snapshot of the mesh cells coordinates was selected as spatial reference, specifically the first one obtained. Then, a subset of $2 \times 10^{6}$ reference cells was chosen at random, in order to reduce the computational cost yet still provide adequate spatial resolution.

For each subsequent snapshot, the coordinates of the cell centroids, that will have changed due to the rotation of the mesh, are inspected to identify amongst them the nearest neighbour of each of the reference coordinates. To optimize the computation, raw coordinates from each new snapshot are firstly organized into a $k-\mathrm{d}$ tree data structure [59]. A $k-d$ tree nearest neighbour searcher algorithm [60] is then used to obtain the indices of the new snapshot cells that best match the reference coordinates, along with their Euclidean distances $d_{i}$ to these reference coordinates.

From these points however, we have excluded those belonging to the impeller, since the blade passing introduces a discontinuity in the cells pressure records that in turn produces spurious results when the decomposition procedure is applied. The filtering out of the impeller region is done by removing cells that have, simultaneously, a height lower than the blades leading edges $\left(z_{i}<z_{\mathrm{LE}}\right)$ and a distance from the axis lower than the wheel outer radius $\left(x_{i}^{2}+y_{i}^{2}<r_{\mathrm{O}}^{2}\right)$.

A final sanity check is then performed to only keep cells whose distance $d_{i}$ to the corresponding reference cell is lower than $1 \mathrm{~mm}$, in order to ensure suitable spatial consistency. Once that all suitable cells of the snapshot are found, their pressure values are stored in the corresponding vector $\mathbf{v}_{i}$. Positions in this vector that failed any of the sanity checks are filled with NaN values.

When the snapshot matrix $\mathbf{V}$ is then assembled, rows with NaN values are culled, thus obtaining a matrix of consistent, continuous pressure records at fixed locations. Note that if a variable time step approach is preferred, a further time domain interpolation would be needed in order to keep the sampling interval constant.

\section{Results and discussion}

Once that the results from the simulation were validated, a preliminary analysis of the raw pressure field was carried out, in order to assess the presence of spinning-mode-like structures. In Fig. 5 the pressure field of the inlet duct near the compressor is represented. Cross-section slices have been

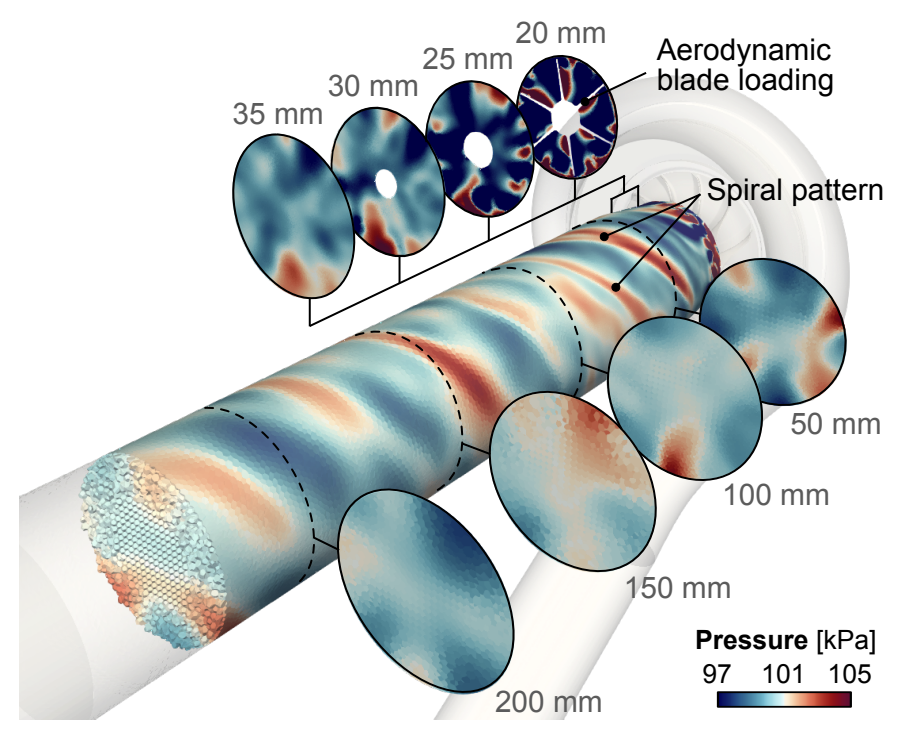

Figure 5: Raw pressure field in the inlet duct, with selected cross-sections highlighted. Each slice is marked with its z coordinate, measured from the bottom of the wheel. 


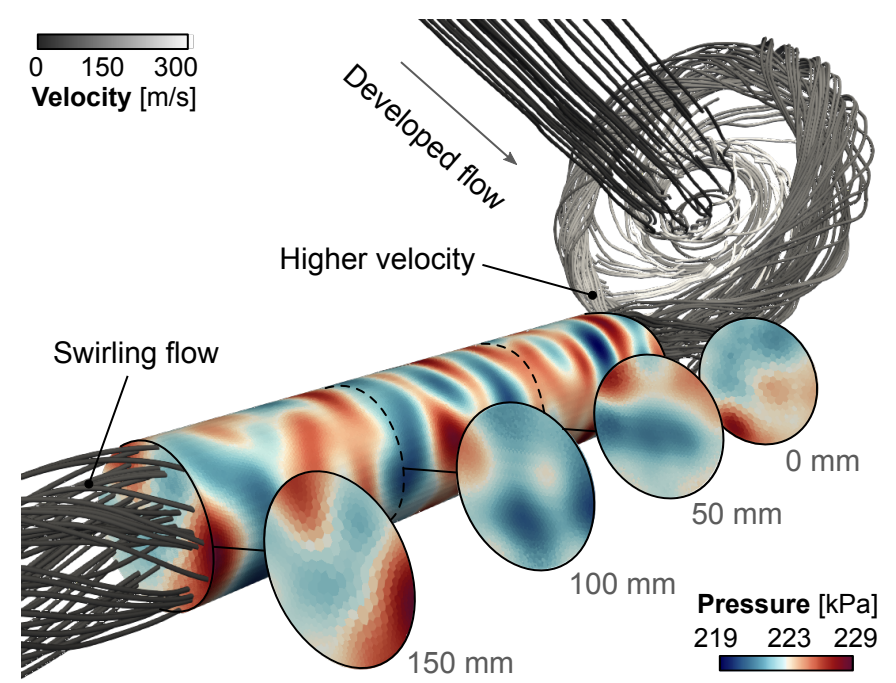

Figure 6: Raw outlet pressure field. Slice positions are measured from the end of the volute. Streamlines shaded by velocity magnitude are included to showcase the difference between inlet and outlet flow development.

extracted at $50 \mathrm{~mm}(1.2 \varnothing)$ intervals, while another set at the top shows slices extracted each $5 \mathrm{~mm}$, with the first one $3 \mathrm{~mm}$ downstream of the leading edges. The impeller hub is also present in the center of the first three of these slices.

While a certain spiral pattern similar to those of the theoretical Tyler-Sofrin modes can be noticed, especially close to the wheel, cross-section pressure patterns along the duct do not bear a close resemblance to the theoretical model discussed in section 2.1. Inspection of these duct slices seems to suggest the presence of three or four maxima and minima at each section, hinting at the propagation of an $m=4$ modal structure, although intermixed with other patterns.

On the other hand, the most downstream slice at the top of the figure shows clearly the aerodynamic loading of the impeller blades themselves. As the operation point is set at the precise onset of flow instabilities, regions of high pressure can be observed along the periphery of the inducer, more pronounced in the center of some of the channels, and consistent with fluid cells of recirculating backflow cells with null axial velocity. However, in the subsequent upstream slices separated just by $5 \mathrm{~mm}$, the distinct pressure pattern of the blades rapidly fades into a less clear one, as many hydrodynamic components cannot propagate.

This lack of clarity in the pressure pattern is exacerbated in the outlet duct, as seen in Fig. 6, where again the pressure fluctuations at the wall and some selected cross-section slices are presented. In this case examination of the oscillations fails to suggest a coherent number of cross-section maxima, nor a clear spiral modal pattern.

It must be taken into account however that in the case of the compressor outlet, theoretical assumptions made during the discussion of the analytical modes are not preserved. In particular, the duct cross section does not have a direct line of sight of the rotating aerodynamic loading of the blades, nor is the flow fully developed.

The later is demonstrated in Fig. 6 by plotting the flow streamlines, coloured by velocity magnitude. While in the inlet the streamlines are parallel indicating a developed flow, it can be seen how the flow is accelerated in the diffuser and then, as it meets the volute, starts a swirling movement.

Velocity of this swirling flow is higher at the start of the volute next to the tongue, where the cross-section is smaller, and then the swirling flow undergoes a deceleration as it travels along the volute and through the outlet duct. Yet, at more than $150 \mathrm{~mm}(3.6 \varnothing)$ from the beginning of the outlet duct, the swirling of the flow is shown to continue.

\subsection{Tyler-Sofrin decomposition}

As a first step towards understanding the structure of sound transmission through the ducts, the classical TylerSofrin modal decomposition was performed, following specifically the method proposed by Banica \& Limacher [21, 22], in which modal distributions $\varphi_{m}\left(r_{j}\right)$ are obtained at discretized

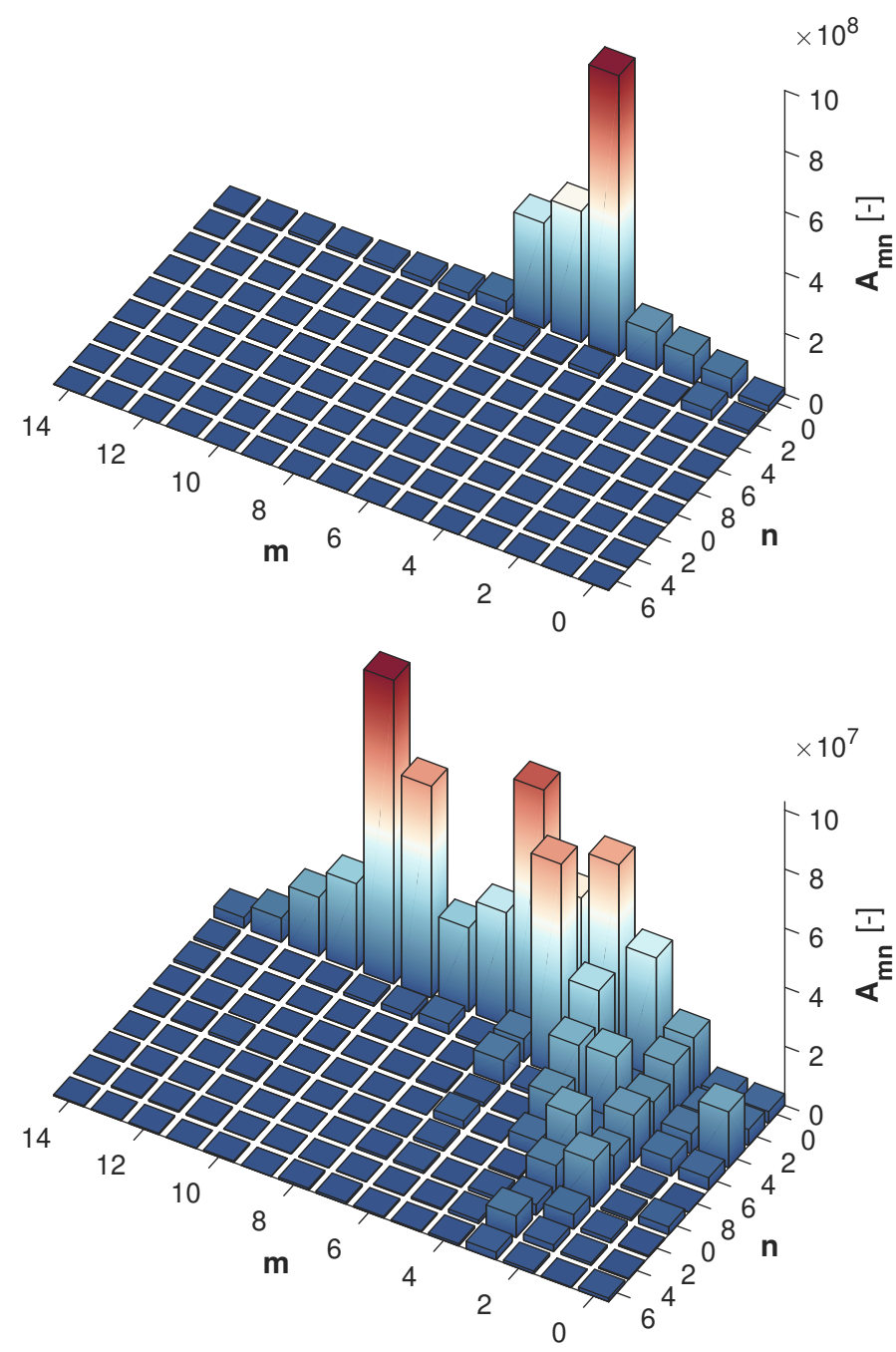

Figure 7: Tyler-Sofrin modal amplitudes $A_{m n}$ reconstructed for both the BPF (top plot) the 2 BPF (bottom plot). 
radial positions $r_{j}$ by means of circumferential Fourier transforms, allowing the least-squares estimation of $A_{m n}$ by:

$$
\varphi_{m}\left(r_{j}\right)=\sum_{n=0}^{N} A_{m n} J_{m}\left(\kappa_{m n} \frac{r_{j}}{R}\right)
$$

Results plotted in Fig. 7 show for instance that clear $(4,0)$ mode comprises most of the BPF amplitude, represented on the top plot. Even thought that one would expect a $m=6$ mode to be dominant due to the number of compressor blades, the cut-off frequency for the particular duct and operating settings is too low to allow the transmission of this mode through the duct.

However, since the axial position of the slice is very close to the blades, it can be seen that the $(5,0)$ and $(6,0)$ modes are still not fully attenuated, indicating that excitation is indeed present with these pressure patterns, even if unable to propagate upstream the compressor. This agrees with the first inspection of the raw pressure field of Fig. 5, particularly focusing on the difference between the first and the last slices.

In the 2 BPF modal amplitudes shown in the bottom plot of Fig. 7 , energy is shared between $(10,0)$ and several other modes. Amplitudes $A_{m n}$ in this case are an order of magnitude lower with respect to the BPF, in agreement with experimental measurements in which the 2 BPF tone was not detected by the inlet sensors, only by the outlet ones. The reason behind such lesser 2 BPF amplitude at the inlet is not immediately evident in light of the information offered by the Tyler-Sofrin decomposition; this will need to be revisited with the aid of the dynamic mode composition.

\subsection{Spectrum of DMD modes}

Following the assembly of the snapshot matrix $\mathbf{V}_{1}^{N}$ from the CFD data, the DMD procedure outlined in section 2.2 was applied. The resulting DMD modes $\boldsymbol{\Phi}_{i}$ were ordered by their associated frequency and ranked using a normalized Kou \& Zhang's criterion. The resulting mode ranking is plotted in Fig. 8, where 'peaking' modes near or above 0.5 in the normalized metric are highlighted.

We have selected six of those prominent DMD modes, covering frequencies between, approximately, 1 and $32 \mathrm{kHz}$. In addition, cut-off frequencies for theoretical $(m, n)$ TylerSofrin mode propagation at both inlet and outlet ducts were calculated and indicated on top of the figure.

Two of the selected modes, $\boldsymbol{\Phi}_{229}$ and $\boldsymbol{\Phi}_{283}$, can be seen to lie inside the plane wave range for both inlet and outlet ducts. The rest of the modes contain the 3D pressure fluctuations; among these the most prominent are clearly related to the BPF at $\sim 16 \mathrm{kHz}\left(\boldsymbol{\Phi}_{117}\right)$ and the second BPF harmonic at $\sim 32 \mathrm{kHz}$ $\left(\boldsymbol{\Phi}_{811}\right)$, matching literature results $[21,61,62]$.

Remarkably, DMD is shown to highlight the importance of the rotating pressure pattern induced aerodynamically by the blades without any previous theoretical assumption or in fact any information about the flow field other than the snapshot matrix V. It can be seen that for the BPF and the $2 \mathrm{BPF}, m_{\max }$ is 4 and 10 respectively in agreement with the Tyler-Sofrin calculation shown in Fig. 7. In this case however, their mode amplitude is equal, unlike with the aforementioned TylerSofrin results at the duct section. This is due to the advantage of DMD being able to consider the whole domain at once, although DMD could be performed with just duct data if only propagating acoustic components were sought.

In addition to the pure BPF modes that previous investigations have focused on, DMD also highlights modal structures at different frequencies that do not respond to a previous theoretical assumption. Apart from the well-known BPF and plane wave modes, some coherent structures are shown to appear at $\sim 11 \mathrm{kHz}\left(\boldsymbol{\Phi}_{147}\right)$ and at $\sim 27 \mathrm{kHz}\left(\boldsymbol{\Phi}_{475}\right)$, signified by modes that stand apart from the rest of the modal spectrum.

Besides the aforementioned significant modes that stand above the DMD spectrum, it can also be observed in Fig. 8 that the significant bulk of the modal energy-shown as grey bars-appears to be concentrated on the frequency range below $30 \mathrm{kHz}$ (this is, below the second harmonic of the BPF)

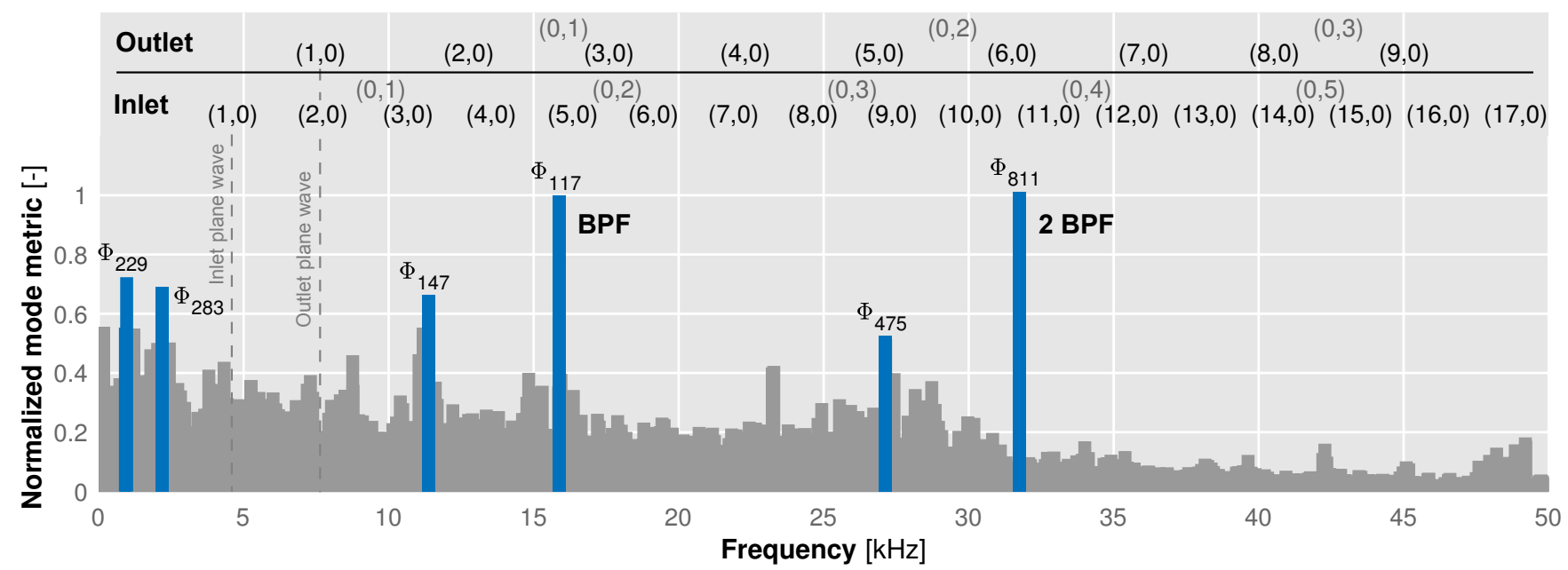

Figure 8: Normalized relevance metric of the DMD modes, highlighting those that will be analyzed in detail, and including the cut-off frequencies at which different Tyler-Sofrin modes start propagating through the inlet and outlet ducts. 
with only some modes slightly peaking near the $50 \mathrm{kHz}$ mark.

Comparing the modes generated automatically by DMD against the theoretical cut-off frequencies for the traditional Tyler-Sofrin modes displayed on top of Fig. 8, it can be seen that despite the BPF excitation pattern, only modes up to $(4,1)$ can be theoretically propagated upstream through the inlet duct, whereas in the outlet case, $(2,0)$ or $(2,1)$ modes could be found. This remarks the added complexity brought by the volute, since transmission of the same excitation pattern must necessarily conform to different cut-off conditions.

Following this line of thought, it is perhaps more telling to consider mode $\boldsymbol{\Phi}_{147}$, containing the flow structures with an excitation frequency of $\sim 11 \mathrm{kHz}$. This mode should only be able to propagate through the outlet duct in the manner of the $(1,0)$ Tyler-Sofrin mode, as cut-in frequencies for the rest of the theoretical modes were higher. In the following subsection however, this hypothesis will be compared against spatial results offered by DMD.

\subsection{Spatial distribution of DMD modes}

As seen in subsection 2.2, the spatial distribution of the energy of flow structures pulsating with a certain frequency $f$ is contained in the $\boldsymbol{\Phi}_{i}(x, y, z)$ values of the DMD mode whose corresponding eigenvalue $\lambda_{i}$ is related with $f$ by Eq. 13. In
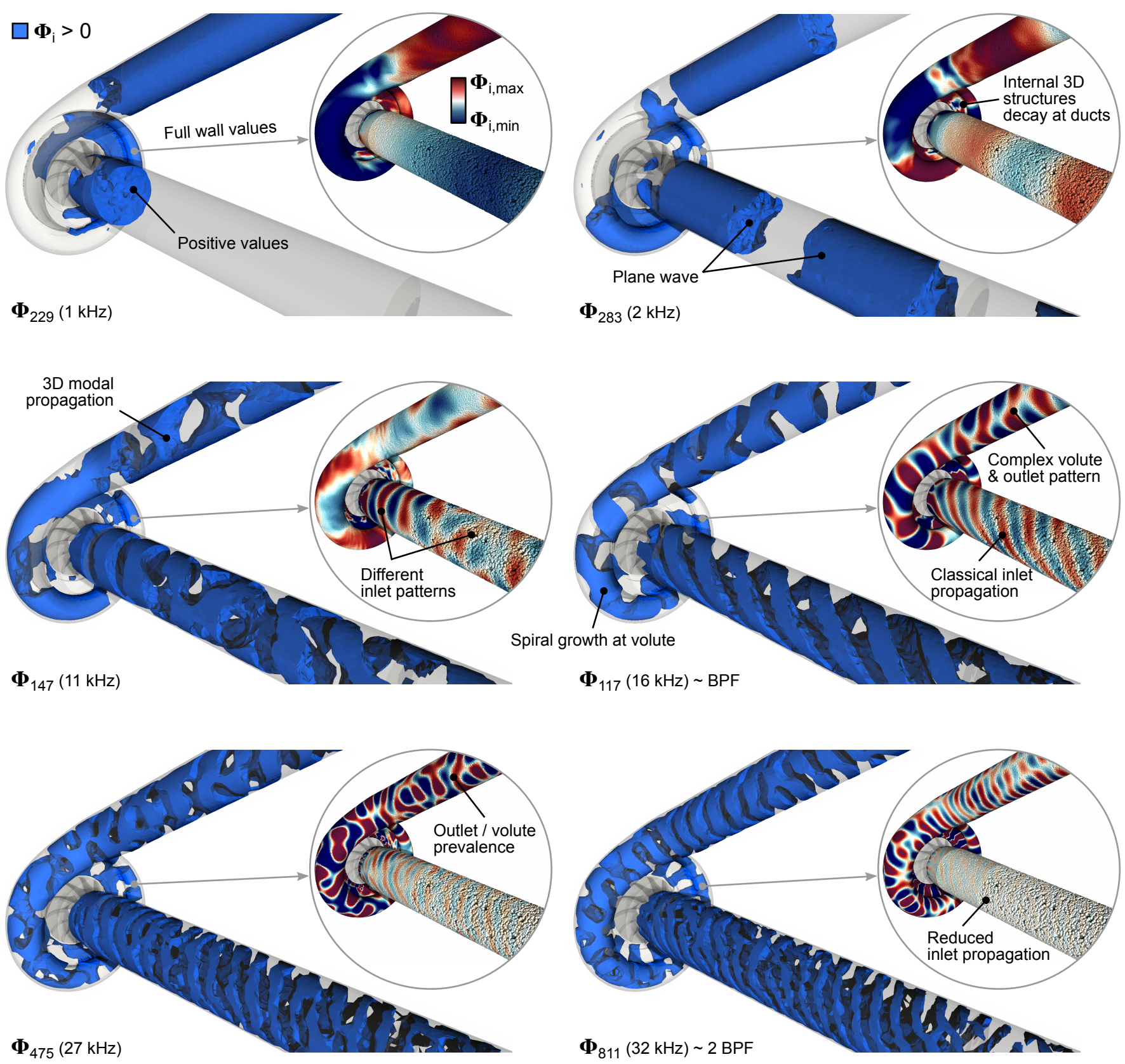

Figure 9: Analysis of selected 3D modal shapes resulting from DMD, with an isovolume of positive values to visualize internal structures and also (in smaller insets) mapping the whole range of values onto a color gradient to show relative spatial amplitude differences. 
Fig. 9 these $\boldsymbol{\Phi}_{i}(x, y, z)$ values are plotted for the most relevant modes, as discussed previously.

Two representations of these values have been chosen. By plotting in uniform color the 3D isovolume of all positive values $\left(\boldsymbol{\Phi}_{i}>0\right)$, the internal structure of each acoustic mode is clearly revealed. However, this visualization technique does not allow for quantification of the attenuation at different locations, hence why each mode in Fig. 9 is accompanied by an inset showing a graded scale of the surface values, from $\min \left(\boldsymbol{\Phi}_{i}\right)$ to $\max \left(\boldsymbol{\Phi}_{i}\right)$, normalized for each mode.

Inspecting the spatial distribution of energy in these selected modes, one can clearly appreciate the plane wave propagation in the inlet and outlet ducts of DMD modes $\boldsymbol{\Phi}_{229}$ and $\boldsymbol{\Phi}_{283}$, which is consistent with the theoretical model. Complex pulsating structures arise in the inducer and the volute, as the hydrodynamic fluctuations there are taken into account by DMD, illustrating thus the hydrodynamic/acoustic splitting.

For higher frequency modes, 3D propagation through the ducts becomes apparent. It is interesting to revisit the previous assumption regarding $\boldsymbol{\Phi}_{147}$ outlet transmission, as it is seen now that a more complex pattern than $(1,0)$ is in effect, highlighting the swirling effect of the volute that impacts the theoretical assumptions. Of note are as well the two different inlet patterns, probably due to the pulsating sources at this fre- quency being established by recirculation bubbles [51] being 'chopped' by the leading edges [63], with a different upstream propagation pattern as imposed by cut-off frequencies outside the recirculation.

This effect is absent from $\boldsymbol{\Phi}_{117}$, which at $\sim 16 \mathrm{kHz}$ gathers the BPF tonal noise distribution. In this case, a classical rotating pattern can be appreciated in the inlet, closely matching for instance those shown in Fig. 1. Inspection of the internal structure however reveals a spiral growth of the pulsating structure as the flow traverses the volute, establishing a more complex pattern in the volute and outlet duct walls.

As the frequency of the flow structures gathered in each mode increases, such as in $\boldsymbol{\Phi}_{475}$ and $\boldsymbol{\Phi}_{811}$ (2 BPF), we can again observe these complex volute and outlet patterns, in contrast with more classical structures in the inlet. However, inlet propagation for these modes appears highly attenuated in comparison with the outlet even if the characteristic spiral structure is still faintly present. This matches the experimental observations described in subsection 3.2, as well as the much lower Tyler-Sofrin modal amplitudes shown in Fig. 7, offering a more comprehensive understanding of the spatial distribution of the $2 \mathrm{BPF}$ pulsation. Yet, it is still not quite clear which is the exact flow mechanism that explains this reduced inlet propagation.
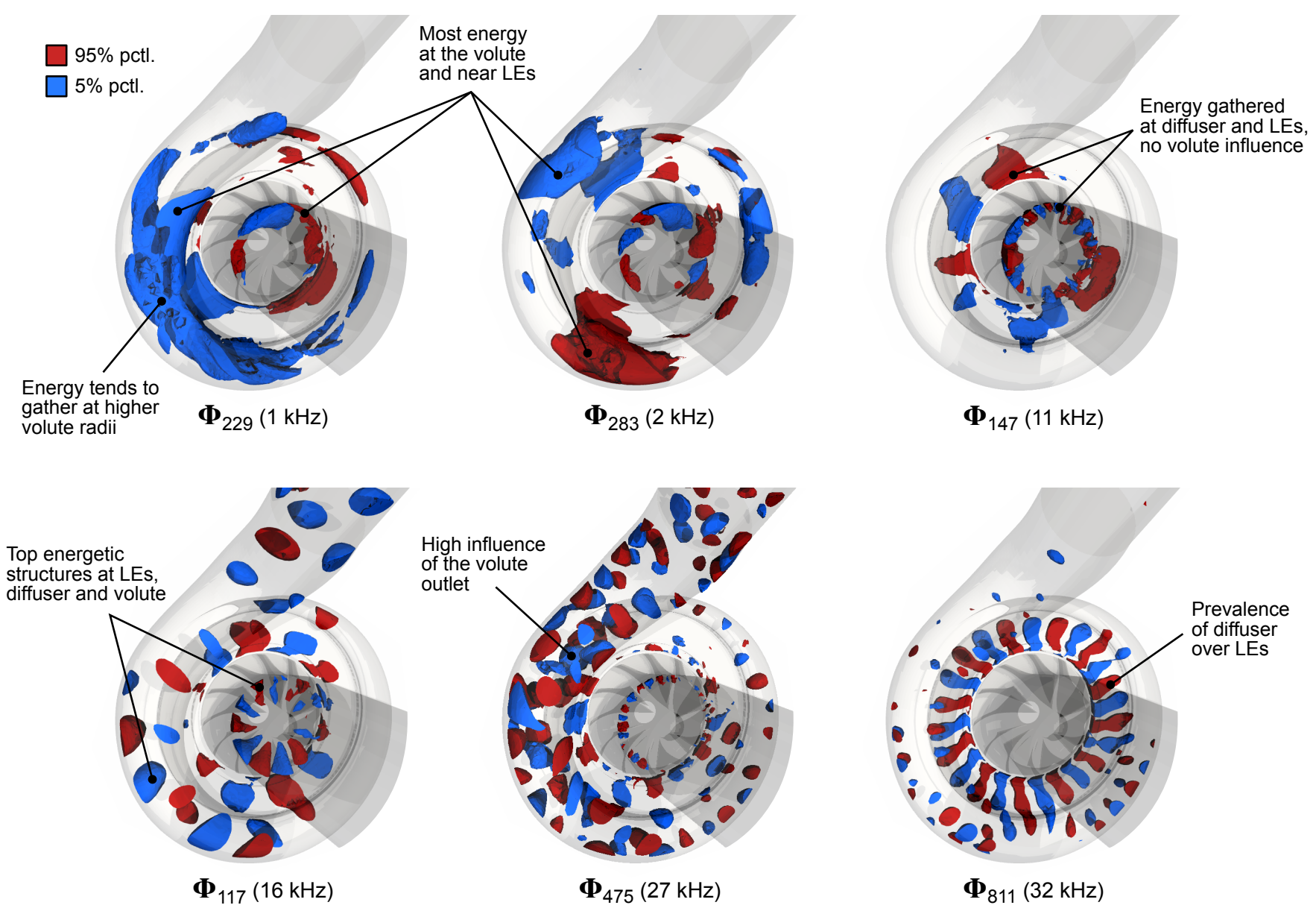

Figure 10: Isovolumes representing percentiles 5\% and 95\% of the spatial energy distribution for the selected DMD modes. 
In order to gain further insight into these flow mechanisms that drive the propagation of the acoustic modes, we can isolate those values among the spatial energy of each mode $\boldsymbol{\Phi}_{i}(x, y, z)$ that account for the $5 \%$ and $95 \%$ percentiles of their distribution. Isovolumes of those values, representing thus highly energetic flow regions pulsating at the different mode frequencies, are shown in Fig. 10.

It can be seen that for modes $\boldsymbol{\Phi}_{229}$ and $\boldsymbol{\Phi}_{283}$ whose characteristic frequency will lead to plane wave propagation, most of the sources are located in the volute and near the blade leading edges. Pulsating energy gathers at higher volute radiialmost the whole volute is pulsating in unison in $\boldsymbol{\Phi}_{229}$ whereas for $\boldsymbol{\Phi}_{283}$ alternating high and low regions are present as the volute grows. This alternation is more pronounced immediately upstream the leading edges; in both modes one side of the wheel presents more pulsating energy than the other, a possible sign of stall inception at a specific location.

On the other hand, the most energetic flow regions for the next highlighted mode, $\boldsymbol{\Phi}_{147}$, are mostly gathered at the diffuser, in large alternating patches, and at the leading edges of the wheel, where around ten cells of opposite amplitude are found. This may be correlated with the frequency of the mode, and seems to indicate a source flow phenomenon related to each channel, including the splitters. No significant influence of the volute is found for this particular mode, in contrast with the others.

Perhaps the most clear mode of Fig. 10 is $\boldsymbol{\Phi}_{117}$, spanning the flow features associated to the BPF frequency. Twelve fluid cells of alternating pressure pulsation are found attached to the blades, in a pattern matching the raw aerodynamic loading depicted in Fig. 5. A similar number of alternating regions is established in the diffuser, with a swirling structure of maxima and minima traversing the higher radii of the volute and continuing orderly along the outlet duct.

A more complex scattering of the modal energy is shown for $\boldsymbol{\Phi}_{475}$; in this case high amplitude fluid cells at the leading edges are much smaller and limited to the outer radius. Similarly, a reduced number of small cells are shown attached to the trailing edges, without spanning across the diffuser. Most of the energetic fluid regions for this frequency are gathered instead at the larger radii of the volute, in coherence with the results from Fig. 9 where the volute and outlet wall amplitudes much more relevant than the inlet's.

Lastly, mode $\boldsymbol{\Phi}_{811}$ shows the distribution of the fluctuating sources associated to the $2 \mathrm{BPF}$. In this case, no high energy regions are detected near the leading edges; most of the energy is established in a clear pattern in the diffuser, where alternating cells are attached in pairs to each trailing edge of the main blades and splitters. This absence of $\sim 32 \mathrm{kHz}$ fluctuations upstream the impeller finally offers a physical explanation for both the lack of amplitude shown in Fig. 9 and the reduced Tyler-Sofrin modal amplitudes evinced in Fig. 7, thus highlighting the usefulness of the DMD technique.

\section{Conclusions}

In this paper, we have analyzed in depth the acoustic field of a radial compressor with volute, by applying different modal decomposition techniques to pressure data generated by a Detached Eddy Simulation of the system operating at the onset of flow instabilities that lead to partially stalled conditions.

On one hand, we have applied the classical Tyler-Sofrin model of acoustic mode propagation through circular ducts. This time-proven technique has the advantage of being experimentally realizable even with a low number of sensors, as demonstrated recently by Limacher et al. [21].

However, it relies on a number of assumptions as to the flow development and homogeneity, the constant geometry of the ducts, the location of the rotating pressure pattern, and so on. In addition, the data-gathering is performed at particular slices, assuming that modal remain constant along the duct. In the specific case of radial compressors, many of those do not hold true, especially with complex ducts and volutes. Thus, elucidating the influence of those complex geometries on the spectral signature of the compressor through the Tyler-Sofrin approach becomes a difficult endeavour.

On the other hand, once that a reliable numerical simulation of the compressor has been obtained, the use of datadriven, assumptions-free modal decomposition techniques such as DMD offers a comprehensive, actionable understanding of the relation between the spatial features of the flow and the acoustic emission at different frequencies. By being able to take into account the pressure information of the whole domain at once, including inside complex geometries such as the diffuser, volute, curved ducts, etc., the overall amplitude for each frequency is better characterized according to the overall pulsating energy available in the flow.

In the specific case of radial compressors with volutes or manifolds, it has been shown in this investigation that for many frequencies the most energetic flow features are confined in the diffuser and volute, where they are not usually taken into account if Tyler-Sofrin techniques are used. In addition, complex pulsating spatial patterns not accounted for with the classical model have been observed, opening the door to specific modifications of the design in order to improve the noise level at those frequencies.

Therefore, it can be concluded that as the geometry of radial compressors becomes more complex due to tighter packaging requirements, miniaturized engines, recirculating cavities, ported shrouds, etc., the in-depth understanding afforded by data-driven approaches such as the one demonstrated in this paper are posed to become an important tool in the quest for quieter, more sustainable engines.

\section{Conflict of interest statement}

None declared. 


\section{Acknowledgements}

The equipment used in this work has been partially supported by the Spanish Ministerio de Economía y Competitividad through grant [DPI2015-70464-R] and by FEDER project funds "Dotación de infraestructuras científico técnicas para el Centro Integral de Mejora Energética y Medioambiental de Sistemas de Transporte (CiMeT), [FEDER-ICTS-2012-06]" framed in the operational program of unique scientific and technical infrastructure of the Spanish Ministerio de Economía y Competitividad. F. Roig is partially supported through the Programa de Apoyo para la Investigación y Desarrollo of the Universitat Politècnica de València [PAID-01-17].

\section{References}

[1] A. Christie, Death in the clouds, Collins Crime Club, 1935.

[2] J. M. Tyler, T. G. Sofrin, Axial flow compressor noise studies, Tech. rep., SAE Technical Paper (1962). doi : 10.4271/620532.

[3] C. Morfey, Rotating pressure patterns in ducts: their generation and transmission, J. Sound Vib. 1 (1) (1964) 60-87. doi:10.1016/ $0022-460 \times(64) 90007-0$.

[4] W. Neise, L. Enghardt, Technology approach to aero engine noise reduction, Aerosp. Sci. Technol. 7 (5) (2003) 352-363. doi: 10.1016/ S1270-9638 (03) 00027- 0 .

[5] D. Casalino, F. Diozzi, R. Sannino, A. Paonessa, Aircraft noise reduction technologies: a bibliographic review, Aerosp. Sci. Technol. 12 (1) (2008) 1-17. doi : 10.1016/j . ast. 2007.10.004.

[6] T. Raitor, W. Neise, Sound generation in centrifugal compressors, J. Sound Vib. 314 (2008) 738 - 756. doi : 10.1016/j . j sv . 2008.01.034.

[7] D. Fink, N. Cumpsty, E. Greitzer, Surge dynamics in a free-spool centrifugal compressor system, J. Turbomach. 114 (2) (1992) 321-332. doi:10.1115/1.2929146.

[8] H. Krain, Review of centrifugal compressor's application and development, J. Turbomach. 127 (1) (2005) 25-34.

[9] I. Day, Stall, surge, and 75 years of research, J. Turbomach. 138 (1) (2016) 011001-1-011001-16. doi:10.1115/1.4031473.

[10] J. Lorett, S. Gopalakrishnan, Interaction between impeller and volute of pumps at off-design conditions, Journal of Fluids Engineering 108 (1) (1986) 12-18. doi: 10.1115/1.3242534.

[11] C. Yang, W. Wang, H. Zhang, C. Yang, Y. Li, Investigation of stall process flow field in transonic centrifugal compressor with volute, Aerosp. Sci. Technol. 81 (2018) 53-64. doi : 10.1016/j .ast.2018.07.047.

[12] Z. Sun, W. Zou, X. Zheng, Instability detection of centrifugal compressors by means of acoustic measurements, Aerosp. Sci. Technol. 82 (2018) 628-635. doi:10.1016/j .ast.2018.09.006.

[13] I. Shahin, M. Alqaradawi, M. Gadala, O. Badr, On the aero acoustic and internal flows structure in a centrifugal compressor with hub side cavity operating at off design condition, Aerosp. Sci. Technol. 60 (2017) 68-83. doi:10.1016/j . ast. 2016.10.031.

[14] S.-B. Ma, K.-Y. Kim, Optimization of discrete cavities in a centrifugal compressor to enhance operating stability, Aerosp. Sci. Technol. 68 (2017) 308-319. doi:10.1016/j .ast.2017.05.029.

[15] X. He, X. Zheng, Roles and mechanisms of casing treatment on different scales of flow instability in high pressure ratio centrifugal compressors, Aerosp. Sci. Technol. 84 (2019) 734-746. doi: 10.1016/j . ast. 2018. 10. 015 .

[16] A. Michalke, On the propagation of sound generated in a pipe of circular cross-section with uniform mean flow, J. Sound Vib. 134 (2) (1989) 203-234. doi : 10 . 1016/0022-460X (89) 90649- 4.

[17] W. Eversman, Theoretical models for duct acoustic propagation and radiation, in: H. H. Hubbard (Ed.), Aeroacoustics of Flight Vehicles: Theory and Practice. (NASA Reference Publication 1258), Vol. 2, NASA, 1991, pp. 101-163.

[18] A. McAlpine, M. Wright, Acoustic scattering by a spliced turbofan inlet duct liner at supersonic fan speeds, J. Sound Vib. 292 (3) (2006) 911934. doi:10.1016/j.jsv.2005.09.010.
[19] L. J. Eriksson, Higher order mode effects in circular ducts and expansion chambers, J. Acoust. Soc. Am. 68 (1980) 545. doi : 10. 1121/1. 384768.

[20] C. Moore, Measurement of radial and circumferential modes in annular and circular fan ducts, J. Sound Vib. 62 (2) (1979) 235-256. doi: 10. 1016/0022-460X(79) 90024- 5 .

[21] P. Limacher, C. Spinder, M. C. Banica, H.-J. Feld, A robust industrial procedure for measuring modal sound fields in the development of radial compressor stages, J. Eng. Gas Turb. Power. 139 (6) (2017) 062604. doi: 10.1115/1.4035287.

[22] M. C. Banica, P. Limacher, H.-J. Feld, C. Spinder, Numerical prediction of the sources and the modal content of the acoustic field in a radial compressor outflow, J. Eng. Gas Turb. Power. 139 (9) (2017) 092605. doi:10.1115/1.4036284.

[23] A. Torregrosa, A. Broatch, X. Margot, J. García-Tíscar, Y. Narvekar, R. Cheung, Local flow measurements in a turbocharger compressor inlet, Exp. Therm. Fluid Sci. 88 (2017) 542-553. doi:10.1016/j . expthermflusci.2017.07.007.

[24] N. Aubry, On the hidden beauty of the proper orthogonal decomposition, Theor. Comp. Fluid Dyn. 2 (5-6) (1991) 339-352. doi:10.1007/ BF00271473.

[25] J. L. Lumley, The structure of inhomogeneous turbulent flows, in: Atmospheric Turbulence and Radio Wave Propagation - Proceedings of the International Colloquium, Nauka, Moscow, 1967, pp. 166-178.

[26] D. Rajpal, E. Gillebaart, R. De Breuker, Preliminary aeroelastic design of composite wings subjected to critical gust loads, Aerosp. Sci. Technol. 85 (2019) 96-112. doi : 10.1016/j .ast.2018.11.051.

[27] J. Luo, Design optimization of the last stage of a 4.5-stage compressor using a pod-based hybrid model, Aerosp. Sci. Technol. 76 (2018) 303314. doi : 10.1016/j .ast. 2018.01.043.

[28] D. Lengani, D. Simoni, R. Pichler, R. Sandberg, V. Michelassi, F. Bertini, Identification and quantification of losses in a lpt cascade by pod applied to les data, Int. J. Heat Fluid Fl. 70 (2018) 28-40. doi:10.1016/j . ijheatfluidflow.2018.01.011.

[29] H. Güner, D. Thomas, G. Dimitriadis, V. Terrapon, Unsteady aerodynamic modeling methodology based on dynamic mode interpolation for transonic flutter calculations, J. Fluid. Struct. 84 (2019) 218-232. doi: 10.1016/j . jfluidst ructs . 2018 . 11.002.

[30] D. Gil-Prieto, P. K. Zachos, D. G. MacManus, G. McLelland, Unsteady characteristics of s-duct intake flow distortion, Aerosp. Sci. Technol. 84 (2019) 938-952. doi:10.1016/j .ast.2018.10.020.

[31] P. J. Schmid, Dynamic mode decomposition of numerical and experimental data, J. Fluid Mech. 656 (2010) 5-28. doi:10.1017/ S0022112010001217.

[32] C. W. Rowley, I. Mezić, S. Bagheri, P. Schlatter, D. S. Henningson, Spectral analysis of nonlinear flows, J. Fluid Mech. 641 (2009) 115127. doi : 10. 1017/S0022112009992059.

[33] K. K. Chen, J. H. Tu, C. W. Rowley, Variants of dynamic mode decomposition: boundary condition, koopman, and fourier analyses, J. Nonlinear Sci. 22 (6) (2012) 887-915. doi : 10. 1007/s00332-012 - 9130 - 9.

[34] N. Vinha, F. Meseguer-Garrido, J. De Vicente, E. Valero, A dynamic mode decomposition of the saturation process in the open cavity flow, Aerosp. Sci. Technol. 52 (2016) 198-206. doi:10.1016/j .ast.2016.02.036.

[35] J. Kou, S. Le Clainche, W. Zhang, A reduced-order model for compressible flows with buffeting condition using higher order dynamic mode decomposition with a mode selection criterion, Phys. Fluids 30 (1) (2018) 016103. doi : 10.1063/1.4999699.

[36] M. Daroukh, S. Moreau, N. Gourdain, J.-F. Boussuge, C. Sensiau, Tonal noise prediction of a modern turbofan engine with large upstream and downstream distortion, J. Turbomach. 141 (2) (2019) 021010-1021010-11. doi: 10.1115/1.4042163.

[37] A. Renaud, S. Tachibana, S. Arase, T. Yokomori, Experimental study of thermo-acoustic instability triggering in a staged liquid fuel combustor using high-speed oh-plif, J. Eng. Gas Turb. Power. 140 (8) (2018) 081505. doi: 10.1115/1.4038915.

[38] D. Papadogiannis, G. Wang, S. Moreau, F. Duchaine, L. Gicquel, F. Nicoud, Assessment of the indirect combustion noise generated in a transonic high-pressure turbine stage, J. Eng. Gas Turb. Power. 138 (4) (2016) 041503. doi:10.1115/1.4031404.

[39] J. Dahan, R. Futrzynski, C. O'Reilly, G. Efraimsson, Aero-acoustic source analysis of landing gear noise via dynamic mode decomposition, in: 21st International Congress on Sound and Vibration, Vol. 2, 2014, 
pp. $1245-1252$.

URL http://WwW.iiav.org/icsv21/content/papers/papers/ full_paper_221_20140402145649114.pdf

[40] E. Sundström, B. Semlitsch, M. Mihaescu, Centrifugal compressor: The sound of surge, in: 21st AIAA/CEAS Aeroacoustics Conference, 2015. doi : 10.2514/6.2015-2674.

[41] B. Semlitsch, M. Mihăescu, Flow phenomena leading to surge in a centrifugal compressor, Energy 103 (2016) 572-587. doi:10.1016/j energy.2016.03.032.

[42] B. O. Koopman, Hamiltonian systems and transformation in hilbert space, Proceedings of the National Academy of Sciences 17 (5) (1931) 315-318.

URL http: //www. pnas . org/content/17/5/315. full.pdf

[43] T. Sayadi, J. Nichols, P. Schmid, M. Jovanovic, Dynamic mode decomposition of h-type transition to turbulence, in: Proceedings of the Summer Program 2012, Center for Turbulence Research, 2012, pp. 5-14.

URL http://people.ece.umn.edu/users/mihailo/papers/ conference/2012/SayadiNicholsSchmidJovanovicCTR2012. pdf

[44] P. J. Schmid, L. Li, M. P. Juniper, O. Pust, Applications of the dynamic mode decomposition, Theor. Comp. Fluid Dyn. 25 (1-4) (2011) 249259. doi : 10. 1007/s00162-010-0203- 9.

[45] S. Bagheri, Koopman-mode decomposition of the cylinder wake, J. Fluid Mech. 726 (2013) 596-623. doi : 10.1017/j fm. 2013. 249.

[46] M. R. Jovanović, P. J. Schmid, J. W. Nichols, Sparsity-promoting dynamic mode decomposition, Phys. Fluids (1994-present) 26 (2) (2014) 024103. doi: 10.1063/1.4863670.

[47] A. Sakowitz, M. Mihaescu, L. Fuchs, Flow decomposition methods applied to the flow in an ic engine manifold, Appl. Therm. Eng. 65 (1) (2014) 57-65. doi:10.1016/j . applthermaleng . 2013 . 12.082.

[48] R. Futrzynski, G. Efraimsson, Dymode: A parallel dynamic mode decomposition software, KTH Royal Institute of Technology (2015). URL http://kth.diva-portal.org/smash/record.jsf?pid= diva2\%3A786623

[49] J. Kou, W. Zhang, An improved criterion to select dominant modes from dynamic mode decomposition, European J. Mech.-B/Fluids 62 (2017) 109-129. doi: 10.1016/j . eu romechflu. 2016.11.015.

[50] A. Broatch, J. Galindo, R. Navarro, J. García-Tíscar, Methodology for experimental validation of a CFD model for predicting noise generation in centrifugal compressors, Int. J. Heat Fluid Fl. 50 (2014) $134-144$. doi: 10.1016/j.ijheatfluidflow.2014.06.006.

[51] A. Broatch, J. Galindo, R. Navarro, J. García-Tíscar, A. Daglish, R. Sharma, Simulations and measurements of automotive turbocharger compressor whoosh noise, Engineering Applications of Computational Fluid Mechanics 9 (1) (2015) 12-20. doi : 10. 1080/19942060. 2015 1004788 .
[52] A. Broatch, J. Galindo, R. Navarro, J. García-Tíscar, Numerical and experimental analysis of automotive turbocharger compressor aeroacoustics at different operating conditions, Int. J. Heat Fluid Fl.doi: 10.1016/j.ijheatfluidflow. 2016.04.003.

[53] F. Mendonça, O. Baris, G. Capon, Simulation of Radial Compressor Aeroacoustics using CFD, in: Proceedings of ASME Turbo Expo 2012, no. GT2012-70028, ASME, 2012, pp. 1823-1832. doi:10.1115/ GT2012 - 70028.

[54] R. Navarro García, A numerical approach for predicting flow-induced acoustics at near-stall conditions in an automotive turbocharger compressor, Ph.D. thesis (2014). doi: 10.4995/Thesis/10251/44114.

[55] J. Serrano, P. Olmeda, F. Arnau, A. Dombrovsky, L. Smith, Methodology to characterize heat transfer phenomena in small automotive turbochargers: Experiments and modelling based analysis, in: ASME Turbo Expo 2014: Turbine Technical Conference and Exposition, American Society of Mechanical Engineers, 2014, pp. 1-13. doi: 10.1115/GT2014-25179.

[56] G. Piñero, L. Vergara, J. M. Desantes, A. Broatch, Estimation of velocity fluctuation in internal combustion engine exhaust systems through beamforming techniques, Measurement Science \& Technology 11 (11) (2000) 1585-1595. doi: 10 . 1088/0957-0233/11/11/307.

[57] A. Hemidi, F. Henry, S. Leclaire, J.-M. Seynhaeve, Y. Bartosiewicz, CFD analysis of a supersonic air ejector. Part I: experimental validation of single-phase and two-phase operation, Appl. Therm. Eng. 29 (89) (2009) 1523 - 1531. doi:10.1016/j . applthermaleng . 2008.07. 003.

[58] P. Welch, The use of fast fourier transform for the estimation of power spectra: a method based on time averaging over short, modified periodograms, Audio and Electroacoustics, IEEE Transactions on 15 (2) (1967) 70-73.

[59] J. L. Bentley, Multidimensional binary search trees used for associative searching, Commun. Acm 18 (9) (1975) 509-517. doi:10.1145/ 361002.361007.

[60] J. H. Friedman, J. L. Bentley, R. A. Finkel, An algorithm for finding best matches in logarithmic expected time, Acm T. Math. Software (TOMS) 3 (3) (1977) 209-226. doi : 10. 1145/355744 . 355745.

[61] A. Zamiri, B. J. Lee, J. T. Chung, Numerical evaluation of transient flow characteristics in a transonic centrifugal compressor with vaned diffuser, Aerosp. Sci. Technol. 70 (2017) 244-256. doi:10.1016/j . ast. 2017.08 .003

[62] M. Witte, B. Torner, F.-H. Wurm, Analysis of unsteady flow structures in a radial turbomachine by using proper orthogonal decomposition, in: ASME Turbo Expo 2018: Turbomachinery Technical Conference and Exposition, Vol. 2A: Turbomachinery, American Society of Mechanical Engineers, Oslo, Norway, 2018. doi : 10.1115/GT2018- 76596.

[63] E. Sundström, B. Semlitsch, M. Mihăescu, Acoustic signature of flow instabilities in radial compressors, J. Sound Vib. 434 (2018) 221-236. doi: 10.1016/j.jsv. 2018.07.040. 